\title{
Reversible posterior leukoencephalopathy syndrome associated with mFOLFOX6 chemotherapy
}

\author{
Mototsugu Matsunaga - Kazuhiro Araki - Keisuke Miwa • \\ Yu Sunakawa - Keishi Yamashita - Masaru Narabayashi · \\ Tsuyoshi Noguchi $\cdot$ Fumio Nagashima Yasutsuna Sasaki
}

Received: 12 January 2012/ Accepted: 16 March 2012/Published online: 21 April 2012

(C) The Japan Society of Clinical Oncology 2012

\begin{abstract}
Reversible posterior leukoencephalopathy syndrome (RPLS) is a serious neurologic disease characterized by visual disturbance, seizures, headache, and altered mental status associated with white matter changes, particularly in the occipital lobes. RPLS has been attributed to chemotherapy, including modified FOLFOX6 regimens consisting of 5-fluorouracil, oxaliplatin, and leucovorin. Although the mechanism of development of RPLS remains unclear, impaired cerebral blood flow autoregulation and endothelial dysfunction seem to be involved. In particular, endothelial dysfunction has been implicated in the pathophysiology of RPLS associated with platinum agents. Platinum-based chemotherapy is thought to have a direct toxic effect on the vascular endothelium, leading to capillary leakage, disruption of the blood-brain barrier, and axonal swelling, triggering vasogenic edema. Much progress has been made in chemotherapy for colorectal cancer, and the increasing use of molecular-targeted agents is expected to further improve the outcome of therapy. RPLS has recently been associated with such molecular-targeted
\end{abstract}

M. Matsunaga ( $\square) \cdot$ K. Araki · K. Miwa · Y. Sunakawa .

K. Yamashita $\cdot$ F. Nagashima $\cdot$ Y. Sasaki

Department of Medical Oncology, International Medical Center, Comprehensive Cancer Center, Saitama Medical University,

1397-1 Yamane, Hidaka, Saitama 350-1298, Japan

e-mail: mmandmacgyver@yahoo.co.jp

M. Narabayashi

Department of Palliative Medicine, International Medical

Center, Comprehensive Cancer Center, Saitama Medical

University, Hidaka, Saitama, Japan

T. Noguchi

Surgical Division, Center for Community Medicine,

Faculty of Medicine, Oita University, Oita, Japan therapy, particularly in patients concurrently receiving platinum agents. This finding is consistent with previous reports suggesting that RPLS occurs more often in patients receiving platinum-based therapy than in those receiving molecular-targeted agents. Because platinum agents are the most widely used in cancer chemotherapy, clinicians should strongly suspect RPLS in patients receiving treatment with this class of drug.

Keywords Reversible posterior leukoencephalopathy syndrome (RPLS) · Modified FOLFOX6 · Platinum . Colorectal cancer

\section{Introduction}

Reversible posterior leukoencephalopathy syndrome (RPLS) is a serious neurological syndrome characterized by visual disturbance, seizures, headache, and altered mental status associated with white matter changes, particularly in the occipital lobes. Although the mechanism underlying RPLS has not been fully elucidated, RPLS reportedly develops during chemotherapy. Chemotherapy for colorectal cancer has progressed markedly, and such cancer now has a relatively good prognosis when used in combination with FOLFOX or FOLFIRI, among other regimens. Additionally, with the increasing use of molecular-targeted agents, for example bevacizumab, cetuximab, and panitumumab, further improved therapeutic outcomes are expected. Current chemotherapy methods are reported to be associated with a variety of adverse events, but reports documenting association of RPLS with chemotherapy for colorectal cancer are relatively rare. We report a case of RPLS caused by mFOLFOX6 chemotherapy for metastatic sigmoid colon cancer. 
Fig. 1 a Cranial MRI obtained on the day of convulsive seizures. b Cranial MRI obtained on the 30th day after convulsive seizures. a FLAIR image. Intense signals can be seen in both occipital lobes. b FLAIR image. The intense signals in both occipital lobes have nearly disappeared
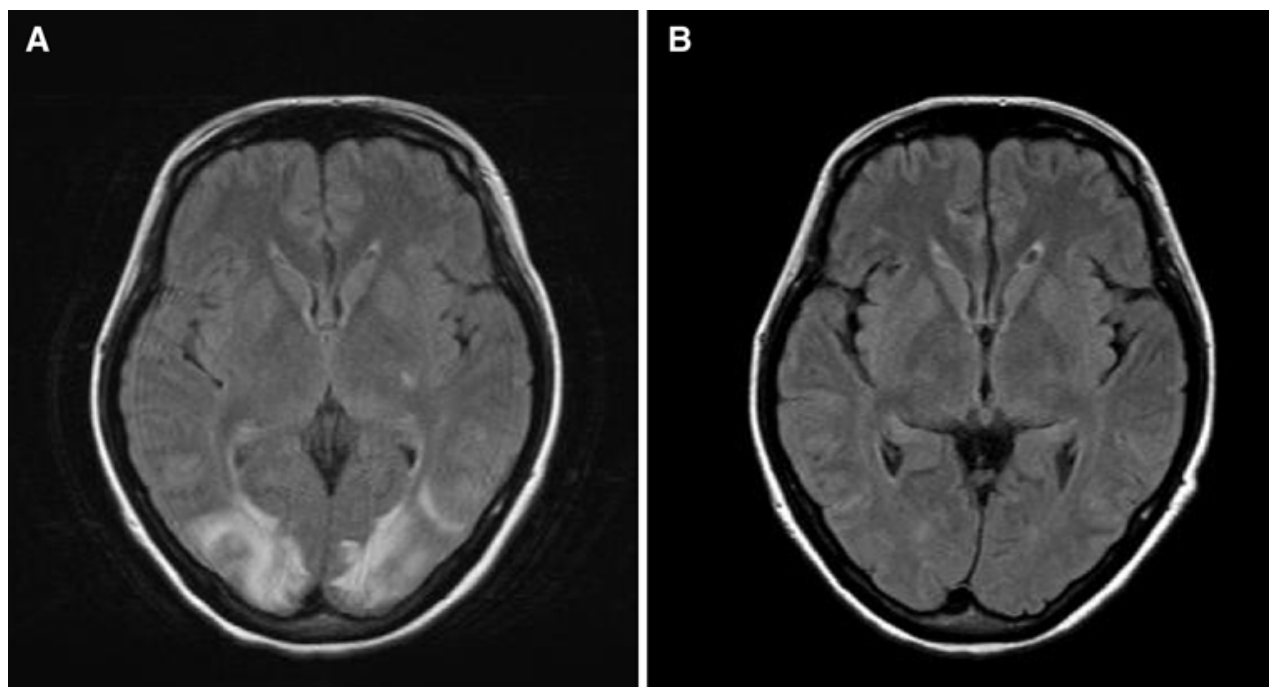

\section{Case report}

The patient was a 43-year-old woman with sigmoid colon cancer and multiple liver metastases. There was no history of epilepsy, renal failure, or hypertension. She was administered mFOLFOX6 with leucovorin $200 \mathrm{mg} / \mathrm{m}^{2}$ as a 2-h infusion and oxaliplatin $85 \mathrm{mg} / \mathrm{m}^{2}$ as a 2-h infusion, followed by bolus 5-fluorouracil $400 \mathrm{mg} / \mathrm{m}^{2}$ and a $46-\mathrm{h}$ infusion of 5 -fluorouracil $2400 \mathrm{mg} / \mathrm{m}^{2}$, repeated every 2 weeks. Early in the morning of day 11, nausea with headache, disturbed consciousness, and visual disturbance developed, and the patient was subsequently transported to the hospital by ambulance. On admission to the emergency room (ER), her vital signs were relatively normal, except for a blood pressure of $177 / 94 \mathrm{mmHg}$. Severe nausea suddenly occurred, and generalized tonic-chronic seizures subsequently developed. Seizures subsided after treatment with diazepam. On the evening of day 11, another episode of generalized tonic-chronic seizures occurred, and the patient received diazepam and phenytoin but did not respond. Status epilepticus developed, and tracheal intubation was performed for airway protection. An intravenous infusion of propofol was administered to control the seizures. Cranial magnetic resonance imaging (MRI) revealed intense signals in both occipital lobes; these were centered at the boundary between the gray matter and white matter on fluid-attenuated inversion recovery (FLAIR) images (Fig. 1a). There was no evidence of metastases, hemorrhage, or infarction in the brain. Hematologic, serum chemical, and cerebrospinal fluid examinations revealed no remarkable changes. Hematologic and blood biochemical tests revealed no inflammation, and the results were negative for a variety of viral markers and antinuclear antibodies. After intubation, her condition stabilized with no seizure recurrence. Although hypertension was present on admission to the ER, blood pressure gradually decreased to the normal range without antihypertensive treatment. On day 18 she was extubated. No neurologic sequelae were observed. Encephaloencephalography did not reveal any particular abnormality. Cranial MRI on day 40 showed that the intense signals in both occipital lobes had nearly disappeared (Fig. 1b). This clinical course and the laboratory/ imaging findings did not seem to be consistent with cerebral infarction, demyelinating disease, angiitis, or encephalitis. Because the patient's condition was characterized by a reversible central nervous system disorder, the possibility of RPLS was considered. Although symptoms of RPLS improved, her colon cancer worsened. The patient died on day 113 without receiving further chemotherapy.

\section{Discussion}

In 1996, Hinchey et al. [1] described 15 patients with a reversible syndrome characterized by headache, disturbed consciousness, seizures, and vision loss associated with predominantly posterior lesions on imaging studies. It is not entirely known why RPLS favors posterior circulation, but it may be because of the relative lack of sympathetic innervation at the level of the arterioles supplied by the vertebrobasilar system compared with that in the anterior circulation [2]. Because the symptoms of these patients resolved markedly or disappeared, this entity was called "RPLS". To date, diagnostic criteria for RPLS remain to be established. The diagnosis is therefore based on the clinical course, imaging findings and the exclusion of brain tumors, cerebral infarction, toxic or metabolic encephalitis, and vasculitis. Although the mechanism of RPLS remains to be fully elucidated, several conditions are apparently of crucial importance in its pathogenesis. Hypertensive 
encephalopathy has been most thoroughly studied clinically and experimentally as a potential cause of RPLS. Sudden elevations in systemic blood pressure exceed the autoregulatory capability of the brain vasculature. Regions of vasodilatation and vasoconstriction develop, particularly in arterial boundary zones, and breakdown of the bloodbrain barrier (BBB) occurs with focal transudation of fluid and petechial hemorrhages [1]. Hypertension is the most common feature associated with RPLS [1]. Thus, clinicians should carefully monitor abnormal blood pressure fluctuations. In patients with cancer, various conditions have been associated with RPLS. Platinum agents, most widely used for cancer chemotherapy, are toxic to the central nervous system [3]. We have summarized the clinical characteristics of 20 patients, including ours, in whom RPLS developed after treatment with platinum-based chemotherapy (Tables 1, 2) [3-21].

According to this review of the literature, development of RPLS is more frequent in women, i.e., 16 out of 20 cases reported, but the relationship of the syndrome with gender remains unclear because there have been reports of cases of overt RPLS associated with non-platinum-containing anticancer drugs or other regimens. RPLS associated with eclampsia has, nevertheless, been reported; the accumulation of fluid often observed during pregnancy may have increased the tendency for brain edema to develop [1]. Time of onset of the syndrome is not evaluable because of its variability with therapy regimen, but it ranged from after the first cycle of chemotherapy to after the 13th cycle. In cases of colorectal cancer, RPLS developed after the first cycle (days 11-12 of Cycle 1) in all patients receiving FOLFOX therapy. However, development of PRLS was in the 4th cycle or thereafter (up to Cycle 10) in patients receiving FOLFOX + Bmab therapy. Recent reports have ascribed this syndrome to molecular-targeted agents such as bevacizumab, sorafenib, and sunitinib, but all 7 patients with RPLS who received molecular-targeted therapy were concurrently administered platinum agents. This finding is consistent with those of previous studies reporting that RPLS is more common in patients receiving platinumbased therapy than in those receiving molecular-targeted agents [4]. All drugs can potentially cause RPLS in individual patients. Clinicians should be aware that not only molecular-targeted therapy but also platinum agents can cause RPLS. Cisplatin reportedly causes vascular disruption, resulting in cerebral vascular accidents, myocardial

Table 1 Clinical characteristics of 20 patients with RPLS after platinum-based chemotherapy

\begin{tabular}{|c|c|c|c|c|c|}
\hline Patient no. & Age (years)/sex & Diagnosis & Regimen & $\begin{array}{l}\text { Highest blood } \\
\text { pressure }(\mathrm{mmHg})\end{array}$ & Refs. \\
\hline 1 & $68 / \mathrm{F}$ & Metastatic colon cancer & FOLFOX + bevacizumab & $140 / 70$ & Peter et al. [5] \\
\hline 2 & $63 / \mathrm{F}$ & Metastatic colon cancer & FOLFOX + bevacizumab & NA & Lau and Paunipagar [6] \\
\hline 3 & $52 / \mathrm{F}$ & Metastatic rectal cancer & FOLFOX + bevacizumab & $172 / 100$ & Ozcan et al. [7] \\
\hline 4 & $62 / \mathrm{F}$ & Metastatic rectal cancer & XELOX + bevacizumab & $190 / 88$ & Pinedo et al. [4] \\
\hline 5 & $54 / \mathrm{F}$ & Gastric cancer & $\begin{array}{l}\text { Cisplatin, capecitabine, } \\
\text { trastuzumab }\end{array}$ & $162 / 114$ & Kaneda et al. [8] \\
\hline 6 & $6 / \mathrm{M}$ & Hepatoblastoma & GEMOX-bevacizumab & $160 / 120$ & Levy et al. [9] \\
\hline 7 & $45 / \mathrm{F}$ & Cholangiocarcinoma & GEMOX-bevacizumab & $194 / 112$ & Chang et al. [10] \\
\hline 8 & $43 / \mathrm{F}$ & Metastatic colon cancer & FOLFOX & $177 / 94$ & This study \\
\hline 9 & $35 / \mathrm{F}$ & Colon cancer & FOLFOX & $167 / 119$ & Nagata et al. [11] \\
\hline 10 & $59 / \mathrm{M}$ & Colon cancer & FOLFOX & $156 / 98$ & Sharief and Perry [12] \\
\hline 11 & $45 / \mathrm{F}$ & Small cell lung cancer & Carboplatin, gemcitabine & $126 / 84$ & Bhatt et al. [13] \\
\hline 12 & $69 / \mathrm{F}$ & Small cell lung cancer & Carboplatin, etoposide & $142 / 78$ & Ryan et al. [14] \\
\hline 13 & $50 / \mathrm{F}$ & Non-small cell lung cancer & GC & $184 / 92$ & Connolly et al. [15] \\
\hline 14 & $53 / \mathrm{F}$ & Non-small cell lung cancer & Carboplatin, gemcitabine & NA & Vieillot et al. [16] \\
\hline 15 & $31 / \mathrm{F}$ & Germ cell tumor of the ovary & PVB & $160 / 100$ & Sueblinvong et al. [17] \\
\hline 16 & $17 / \mathrm{F}$ & Germ cell tumor of the ovary & PVB & $160 / 110$ & Manchana et al. [18] \\
\hline 17 & $64 / \mathrm{F}$ & Fallopian tube cancer & $\begin{array}{l}\text { Intravenous paclitaxel } \\
\text { and intraperitoneal cisplatin }\end{array}$ & $176 / 96$ & Onujiogu et al. [19] \\
\hline 18 & $50 / \mathrm{M}$ & Bladder cancer & GC & a & Maeda et al. [20] \\
\hline 19 & $7 / \mathrm{F}$ & Pituitary germ cell tumor & BEP & $158 / 100$ & Belaramani et al. [21] \\
\hline 20 & $70 / \mathrm{M}$ & Osteosarcoma & Cisplatin (intra-arterial injection) & $180 / 100$ & Ito et al. [3] \\
\hline
\end{tabular}

FOLFOX 5-fluorouracil, leucovorin, oxaliplatin, XELOX capecitabine, leucovorin, oxaliplatin, GEMOX gemcitabine, oxaliplatin, GC gemcitabine, cisplatin, $P V B$ cisplatin, bleomycin, vinblastine, $B E P$ cisplatin, etoposide, bleomycin, $N A$ not available

${ }^{a}$ Nearly normal throughout the entire clinical course 
Table 2 Summary of clinical characteristics of 20 patients with RPLS after platinum-based chemotherapy

\begin{tabular}{|c|c|}
\hline Number of patients & $N=20$ \\
\hline \multicolumn{2}{|l|}{ Age (years) } \\
\hline Median & 47 \\
\hline Range & $6-70$ \\
\hline \multicolumn{2}{|l|}{ Sex } \\
\hline Male & 4 \\
\hline Female & 16 \\
\hline \multicolumn{2}{|l|}{ Regimen } \\
\hline Molecular-targeted agent-based regimen & 7 \\
\hline Bevacizumab-based regimen & 6 \\
\hline Trastuzumab-based regimen & 1 \\
\hline Cisplatin-based regimen & 1 \\
\hline Carboplatin-based regimen & 0 \\
\hline Oxaliplatin-based regimen & 6 \\
\hline $\begin{array}{l}\text { Platinum-based regimen without } \\
\text { molecular-targeted agents }\end{array}$ & 13 \\
\hline Cisplatin-based regimen & 7 \\
\hline Carboplatin-based regimen & 3 \\
\hline Oxaliplatin-based regimen & 3 \\
\hline \multicolumn{2}{|l|}{ Type of cancer } \\
\hline Colorectal & 7 \\
\hline Lung & 4 \\
\hline Liver & 2 \\
\hline Ovary & 2 \\
\hline Bone & 1 \\
\hline Fallopian tube & 1 \\
\hline Gastric & 1 \\
\hline Pituitary & 1 \\
\hline Urologic & 1 \\
\hline \multicolumn{2}{|l|}{ Blood pressure (systolic/diastolic) (mmHg) } \\
\hline Median & $165 / 98$ \\
\hline Range & $126-194 / 70-120$ \\
\hline
\end{tabular}

infarction, and Raynaud's syndrome [22]. Platinum may have direct toxic effects on vascular endothelial cells, similarly to agents that cause vascular disruption [23], which potentially leads to abnormal fluctuations in blood pressure and breakdown of the BBB. Although the possibility that RPLS might have developed as a result of the vascular endothelial toxicity of 5-fluorouracil cannot be completely ruled out, the cerebral toxicity of 5-fluorouracil is well-described as a multifocal inflammatory leukoencephalopathy. Multifocal inflammatory leukoencephalopathy typically develops 6 weeks to 5 months after the initiation of chemotherapy, and is a clinically and radiographically distinct syndrome from RPLS, making it unlikely that 5-fluorouracil was the cause of the RPLS in this patient $[24,25]$. Therefore, we hypothesize that platinum- induced vasculoendothelial damage in our patient led to RPLS development. Given the recent increases in the prevalence of colorectal cancer, clinicians should recognize RPLS as a potential complication related to treatment with platinum agents and molecular-targeted therapy.

Conflict of interest The authors declare no conflict of interest.

\section{References}

1. Hinchey J, Chaves C, Appignani B et al (1996) A reversible posterior leukoencephalopathy syndrome. N Engl J Med 334: 494-500

2. McKinney AM, Short J, Truwit CJ et al (2007) Posterior reversible leukoencephalopathy syndrome: incidence of atypical regions of involvement and imaging findings. AJR Am $J$ Roentgenol 189:904-912

3. Ito Y, Arahata Y, Goto Y et al (1998) Cisplatin neurotoxicity presenting as reversible posterior leukoencephalopathy syndrome. Am J Neuroradiol 19:415-417

4. Pinedo DM, Shah-Khan F, Shah PC (2007) Reversible posterior leukoencephalopathy syndrome associated with oxaliplatin. J Clin Oncol 25:5320-5321

5. Peter S, Hausmann N, Schuster A et al (2007) Reversible posterior leukoencephalopathy syndrome and intravenous bevacizumab. Clin Exp Ophthalmol 36:94-96

6. Lau PC, Paunipagar B (2011) Posterior reversible encephalopathy syndrome with bevacizumab. Hong Kong Med J 17:80-81

7. Ozcan C, Wong SJ, Hari P (2006) Reversible posterior leukoencephalopathy syndrome and bevacizumab. N Engl J Med 354:980-982

8. Kaneda H, Okamoto I, Satoh T et al (2011) Reversible posterior leukoencephalopathy syndrome and trastuzumab. Invest New Drugs (Epub ahead of print)

9. Levy CF, Oo KZ, Firema F et al (2008) Reversible posterior leukoencephalopathy syndrome in a child treated with bevacizumab. Pediatr Blood Cancer 52:669-671

10. Chang Y, Mbeo G, Littman SJ (2011) Reversible posterior leukoencephalopathy syndrome associated with concurrent bevacizumab, gemcitabine, and oxaliplatin for cholangiocarcinoma. J Gastrointest Cancer (Epub ahead of print)

11. Nagata Y, Omuro Y, Shimoyama T et al (2009) A case of colon cancer with reversible posterior leukoencephalopathy syndrome following 5-FU and oxaliplatin (FOLFOX regime). Jpn J Cancer Chemother 36:1163-1166

12. Sharief U, Perry DJ (2009) Delayed reversible posterior encephalopathy syndrome following chemotherapy with oxaliplatin. Clin Colorectal Cancer 8:163-165

13. Bhatt A, Farooq MU, Majid A et al (2009) Chemotherapy-related posterior reversible leukoencephalopathy syndrome. Nat Clin Pract Neurol 5:163-169

14. Ryan SA, MacEneaney P, O'Reilly SP et al (2011) Reversible posterior leukoencephalopathy induced by carboplatin and etoposide. Med Oncol (Epub ahead of print)

15. Connolly RM, Doherty CP, Beddy P et al (2007) Chemotherapy induced reversible posterior leukoencephalopathy syndrome. Lung Cancer 56:459-463

16. Vieillot S, Pouessel D, de Champfleur NM et al (2007) Reversible posterior leukoencephalopathy syndrome after carboplatin. Ann Oncol 18:608-609

17. Sueblinvong T, Noophun P, Pataradool K et al (2002) Posterior leukoencephalopathy following cisplatin, bleomycin and 
vinblastine therapy for germ cell tumor of the ovary. J Obstet Gynaecol Res 28:99-103

18. Manchana T, Sirisabya N, Lertkhachonsuk R et al (2006) Transient cortical blindness during chemotherapy (PVB) for ovarian germ cell tumor. J Med Assoc Thai 89:1265-1268

19. Onujiogu N, Lengyel E, Yamada SD (2008) Reversible posterior leukoencephalopathy syndrome following intravenous paclitaxel and intraperitoneal cisplatin chemotherapy for fallopian tube cancer. Gynecol Oncol 111:537-539

20. Maeda T, Kikuchi F, Matsumoto K et al (2010) Gemcitabine and cisplatin chemotherapy induced reversible posterior leukoencephalopathy syndrome in a bladder cancer patient. Int J Clin Oncol 15:508-511

21. Belaramani KM, Lai V, Li CH et al (2011) Reversible posterior leukoencephalopathy syndrome in Chinese children induced by chemotherapy: a review of five cases. Hong Kong Med J 17:61-66
22. Icli F, Karaoguz H, Dincol D et al (1993) Severe vascular toxicity associated with cisplatin-based chemotherapy. Cancer 72: 587-593

23. Nuver J, De Haas EC, Van Zweeden M et al (2010) Vascular damage in testicular cancer patients: a study on endothelial activation by bleomycin and cisplatin in vitro. Oncol Rep 23:247-253

24. Posner JB (1997) Neurotoxicity of 5-fluorouracil and levamisole. Neurol Netw Comment 1:234-239

25. Hook CC, Kimmel DW, Kvols LK et al (1992) Multifocal inflammatory leukoencephalopathy with 5-fluorouracil and levamisole. Ann Neurol 31:262-267 